\title{
USE AN OPEN SOURCE CHANGE MATTERS SITE TO MEASURE THE VEGETATION INDEX IN THE WESTERN PLATEAU OF ANBAR IN IRAQ
}

\author{
Ahmed ALKUBAISI $\mathbb{B D}^{*}$ \\ Ministry of Education, Anbar Education Directorate, Fallujah Education, Fallujah, Iraq
}

Received 28 May 2019; accepted 27 February 2020

\begin{abstract}
As a result of the development of geo-technologies, in recent years, software has emerged that has provided the possibility of deriving information. The most important of these are geographic information systems (GIS), which are many sources in satellite data from multi-spectral satellites, The source via the Internet is one of the most important modern means of providing large data that varied between maps and satellite images (LANDSAT-SPOT-NASA).

The study of land cover changes is one of the most important subjects of interest to the sciences and in particular the geographical research related to the environment and its effects on humans and living organisms. In the current research, the data of interactive open source maps were adopted, which provided the possibility of analysis.

Using the Change Matters esri, it is one of the most efficient locations for detection of land cover changes by dealing with visuals for different periods of time. The study also reviewed some of the open source websites dealing with GIS to indicate their importance and role in the analysis and production of maps. The research aims to employ open-source digital technologies via the web to extract information about land cover changes.
\end{abstract}

Keywords: geographic information systems, open source sites, vegetation cover, spatial analysis.

\section{Introduction}

In simple terms, Open Data means the kind of data which is open for anyone and everyone for access, modification, reuse, and sharing. Open Data derives its base from various "open movements" such as open source, open hardware, open government, open science etc. Open data is important because the world has grown increasingly data-driven, We live in the information age. We get blasted by truckloads of information each day. In terms of free GIS data sources, it seems never-ending. Landsat is the longest continuously running earth monitoring system, collecting millions of multitemporal, multispectral scenes covering the entire planet. Landsat data still remains the best way to understand global land cover, providing a window on earth changes. People use Landsat because it is more efficient than any other technology at meeting the same decision, The three-panel "ChangeMatters" application runs in any Web browser. Users of ESRI's ChangeMatters online tool can compare side-by-side images of a specific are taken decades apart to se the changes apparent in infrared imagery or in the visible portion of the electromagnetic spectrum. Pan and zoom around the maps to understand earth changes that have happened over time. Advanced change detection tools are also available by clicking any full screen button. The research concluded that the use of modern technologies via the web through which digital information can be derived is important.

\section{Research importance:}

The importance of research comes through the introduction of advanced technical method in dealing with open-source space data and interactive maps through the Internet to monitor the changes of land cover of the search area using GIS or WEB LATER.

\section{Search aim:}

The purpose of the research is to review the practical steps for using the open-source interactive data maps through the Internet and GIS sites to monitor and detect the changes in land cover in the research area for the period $1990-2010$ and to identify the rates of change and ways to address them.

\section{Research problem:}

The problem of research is how to deal with spatial data and interactive maps via the web, and collect digital information to detect land cover changes? Based on the use of open GIS technology, for a different time period.

${ }^{*}$ Corresponding author. E-mail: dr.ahmedm.jihad@gmail.com 


\section{Research methodology:}

The research adopted the method of technical analysis in open source GIS to collect, interpret and extrapolate information to derive maps of land cover changes for the research area. And practical application in websites.

\section{Search area:}

The boundaries of the research area within the province of Anbar, which includes part of the districts of Hit and Haditha and Al-Qa'im, are from the western plateau with an area of $20618 \mathrm{~km}^{2}$. It is characterized by elevation from the east to the west and south-west with a maximum height of 900 meters, The rainfall is often fluctuating and thus the flow of water through the wadis is limited to the duration of rainfall. Look at map (Figure 1) representing the search area.

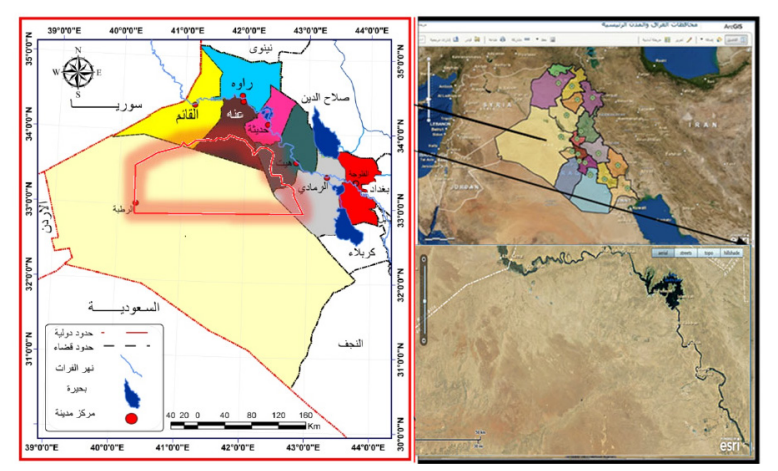

Figure 1. Geographical location of the search area

\section{Materials and methods}

\subsection{The concept of open-source space data}

Download Universal Maps is a tool that helps you get small images of tiles, maps GOOGLE, YAHOO, maps Bing, and maps OpenStreet. All small images are saved and uploaded to the computer, maps can be viewed and uploaded by Map Viewer, or can be combined into a map TIFF-BMP-JPG (esri.com, 2012).

The Open Source Interactive Maps GIS is a technology that allows the user to exchange information across networks without regard to their own format. The development of this technology has led to the necessity of having several basic requirements for the establishment of an open-source interactive geographic mapping system (Al-Din, 2008, p. 90).

\subsection{A stable operating environment}

A shared data space that allows users to complete remote data analysis.A system for browsing data on the Internet dealing with geographical data.

Open-source data (interactive maps) uses Extensible Mark-up Language XML, a simple language written through a text format designed for electronic publishing over the Internet, and then developed into the Marker Language Mark GML Mark-up Language Geography (AlDin, 2008, p. 91).
The mission of the GIS is to provide a spatial database of all the natural, human and economic resources needed by the service systems in the country related to e-government in order to provide different services in line with the real spatial data, which facilitates the direct benefit of the population and supports the decision makers on development programs, Integrated GIS, in other words, Open GIS (Al-Khuzami, 2007, p. 463).

Open source interactive maps are also available within the Map Server by sharing spatial information stored in digital format. It can handle both raster and data. More than 20 vector data formats, including shapefiles, and engineering PostGIS ArcSDE, OpeNDAP, and TIGER files can be submitted (Kropla, 2005, p. xxv).

CIGIS is a web-based support system that facilitates discussion and collaboration in the decision-making process. Allows users, such as technicians, planners and politicians interactively to obtain and share information at various levels, metrics, aspects and details. It also facilitates the cooperation of these users in problem solving throughout the community planning process (Wise \& Craglia, 2008, p. 350). The definition of open source information systems can be summarized by the following (arabspatial.org, n.d.):

1. Freedom of redistribution of the program.

2. Availability of the source text of the program and the free distribution of the source text.

3. The freedom to produce derivative or modified software from the original program, and the freedom to distribute it under the same license for the original software.

4. The license may prevent the distribution of the source text of the modified versions on the condition that the distribution of the files containing the modifications is permitted next to the original text.

5. There is no discrimination in the licensing of any group or persons.

6. There is no definition of program use areas.

7. The rights in the license must be given to whoever is distributed to the program.

These basic features of any license can be called an open source license.

Open GIS Data Warehouse, provides free spatial data as well as spatial data that can be purchased. The website gives a clear right from the start that the user is skilled in GIS. Data sets may be downloaded for specific charges as well as data produced by multimedia media (DeMers, 2009, p. 336).

\section{The most important programs and websites open source}

Universal Maps DownloaderV.5.95 Figure 2, produced by SOFTONPC, specialized in modern systems software and remote sensing, has the advantage of downloading space data from the WWW to any place in the world (advanceduninstaller.com, 2008).

GMap Catcher 0.7.6.1 produced by GNU GENERAL Boston - United States of America (USGS, n.d.). 


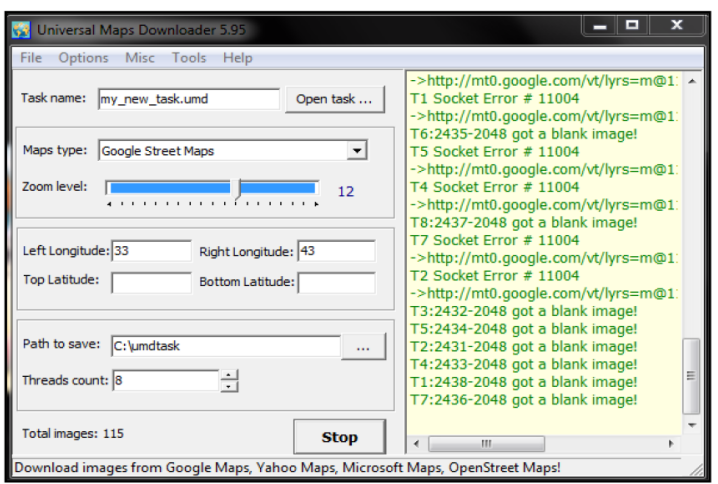

Figure 2. Universal Maps DownloaderV.5.95, produced by SOFTONPC

(LANDSAT-5-7-8), SPOT, and NASA (NASA, n.d.). It is easy to use and select any region in the world by geographic coordinates or UTM (gnu.org, 2009), (Figure 3).

Interactive website ArcGis On Line from the global company esri in cooperation with other companies specialized in modern geotechnical techniques or water engineering. It is easy to use and navigate between the global maps and the possibility of drawing the map and data analysis and participation on the Internet (www.)

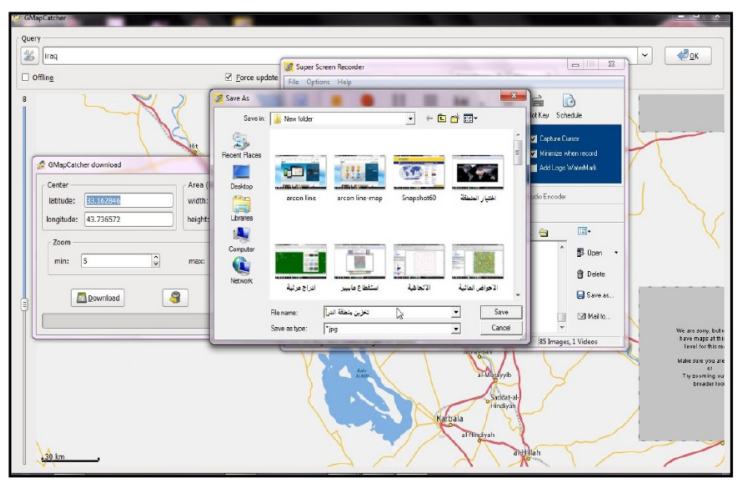

Figure 3. GMap Catcher 0.7.6.1 produced by GNU GENERAL Boston - United States

(esri.com, 2012), with the possibility of reviewing different types of maps Figure 4.

The ChangeMatters.esri website, one of the sites producing GIS and remote sensing techniques designed by Esri International, which has the advantage of loading space data for different periods of time, as well as the possibility of modifying the characteristics of the map according to the search variables, Processing, collection, integration and analysis of data (ChangeMatters, 2013), Figure 5.

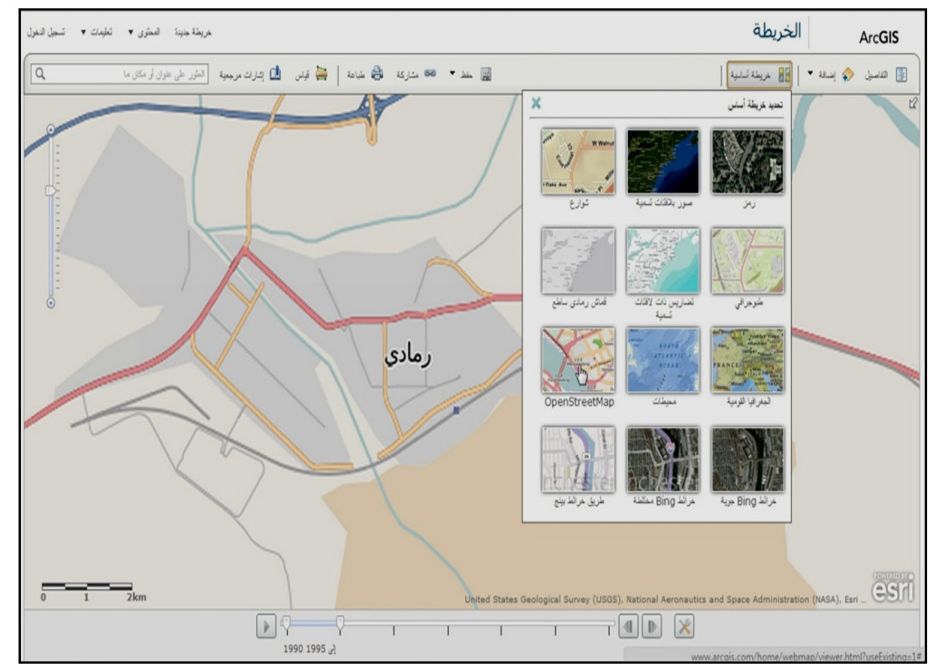

Figure 4. Data analysis and participation on the Internet

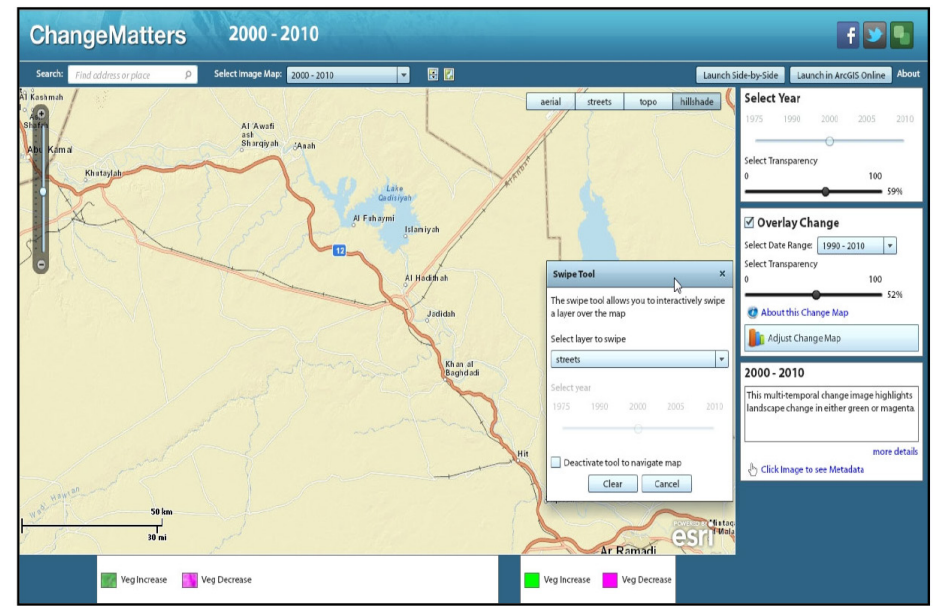

Figure 5. The characteristics of the map according to the search variables 


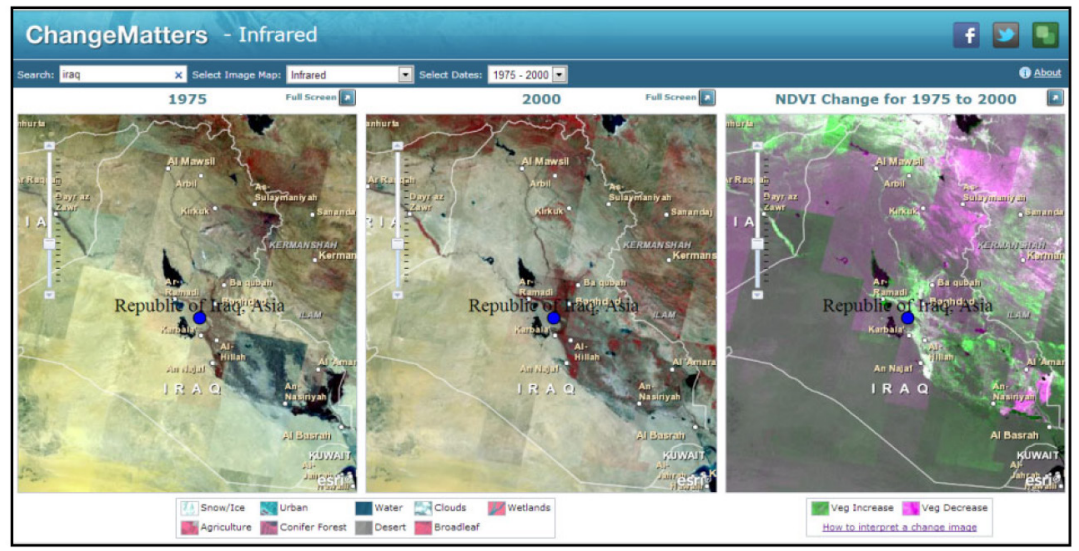

Figure 6. Represents the main interface of the site

\section{Practical application of the research area}

In the present research, the spatial data of the website (http://changematters.esri.com/compare), which has been explained above, has been adopted. This program provides the spatial data of Landsat-7 for five different periods of time 1975-1990. The first represents a first period of time 1975 , the second represents a second time period 1990, and the third represents the produced map after being merged directly into the work. It includes a key Map To read the variables, Figure 6 represents the main interface of the site.

The period of time 1990-2010 was chosen to monitor land cover changes for the research area and to conduct the monitoring and processing of maps and integrate them through the following steps.

\subsection{Duration 1990-2000}

1. Open the website through the link of the program, to show the main window we choose the geographical location of Iraq Figure 7, and then choose the period 19902000 from the window years, then choose from the window Select Image Map.

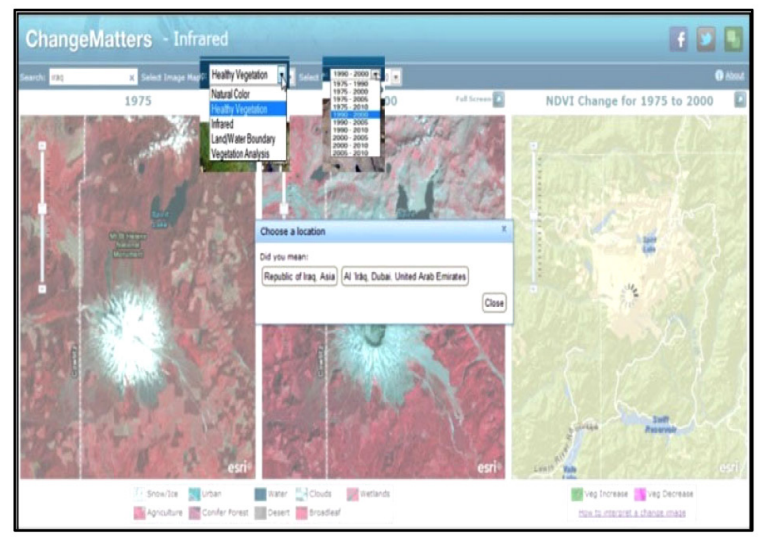

Figure 7. The main window we choose the geographical location of Iraq

2. We define the search area after the command zoom in - zoom out. Due to the nature of the dynamic control of the site through the command zoom in.
3. The program analyzes the map and integrates it with a new map representing see in Figure 8 the level of change through a new key that determines the color of the size of that change.

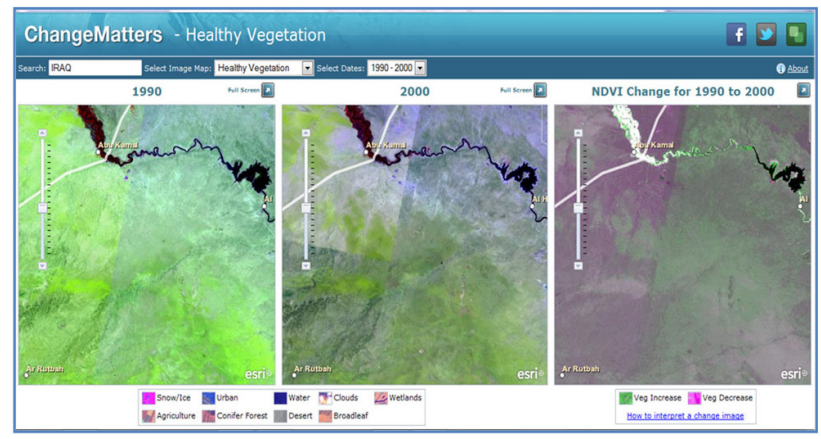

Figure 8 . The program analyzes the map and integrates it with a new map

4. The new interactive map of the time period 19902000 was called in order to classify it and monitor the area and percentages of change in the area through the view full screen, which shows the density of the earth cover according to NDVI equation (Figure 9).

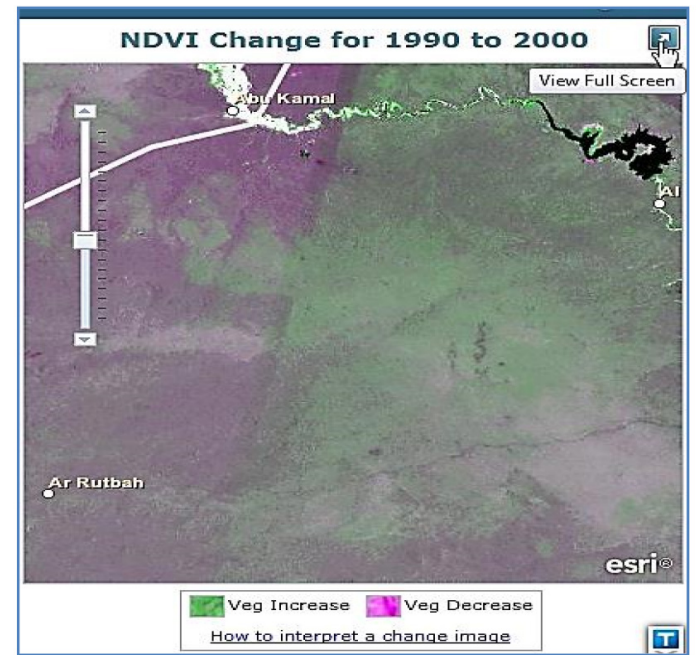

Figure 9. The view full screen, which shows the density of the earth cover according to NDVI 


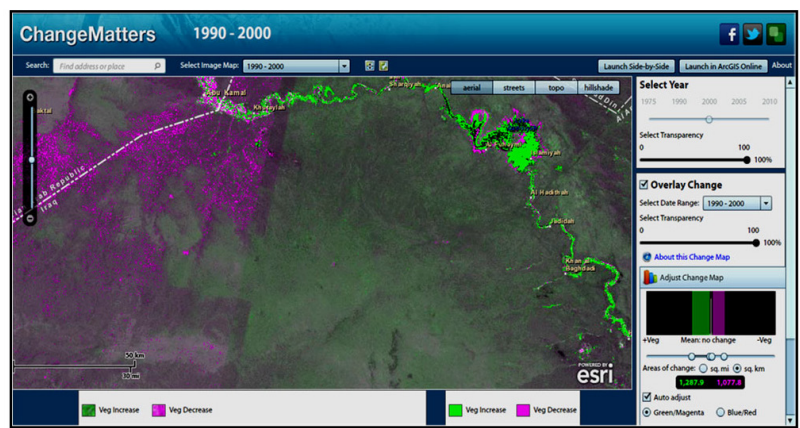

Figure 10. Increasing or decreasing, with the unit measuring the area of change $\mathrm{km}^{2}$ or incl.2

5. From the previous window shows us a new window of the characteristics of the map, which is monitored the size of the change in two colors green indicates the increase in the area $1077 \mathrm{~km}^{2}$, by $5.2236 \%$ and the color purple evidence of the decrease amounted to 12876.2421 . We have shown a number of changes that highlight the temporal change of land cover (vegetation and water)that is see in Figure 10, by increasing or decreasing, with the unit measuring the area of change $\mathrm{km}^{2}$ or incl.2.

6 . The web site provides the basic information window for the concept of difference, analysis and integration of maps, showing the idea of matching spatial data, the values of cells, how they work and the method of analysis

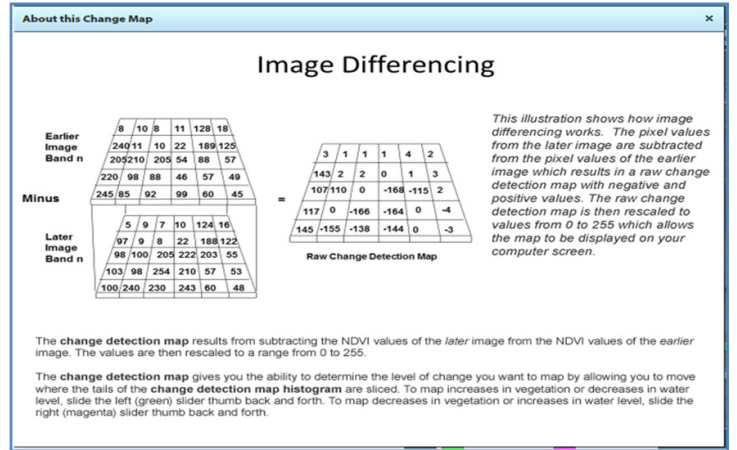

Figure 11. Showing the idea of matching spatial data, the values of cells

according to the density of the earth cover density NDVI (Figure 11).

\subsection{The period of time $2000-2010$}

1. The previous steps are repeated with the chosen time period 2000-2010.

2. The three map 3, which represent the land cover changes of the research area during the period 2000-2010, were analyzed and integrated. Figure 12, which produced the combined map according to the classification of the equation $n d v i$ in two colors purple represents the low area of land cover, and the color green represents the amount of increase in the size of its area (Figure 13).

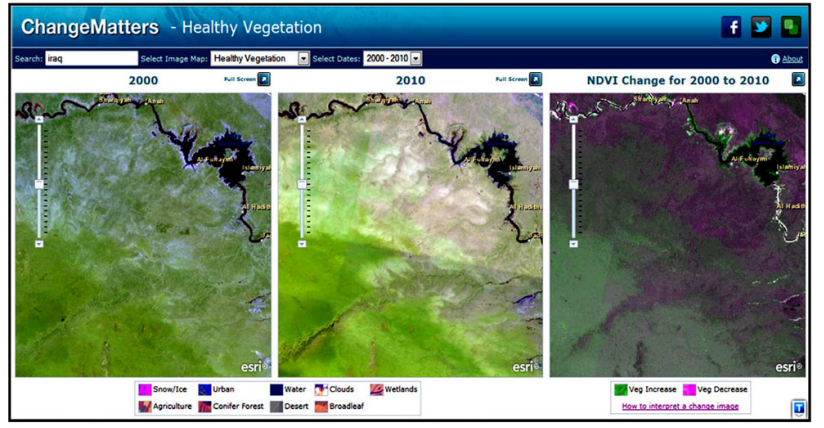

Figure 12. The land cover changes of the research area during the period 2000-2010

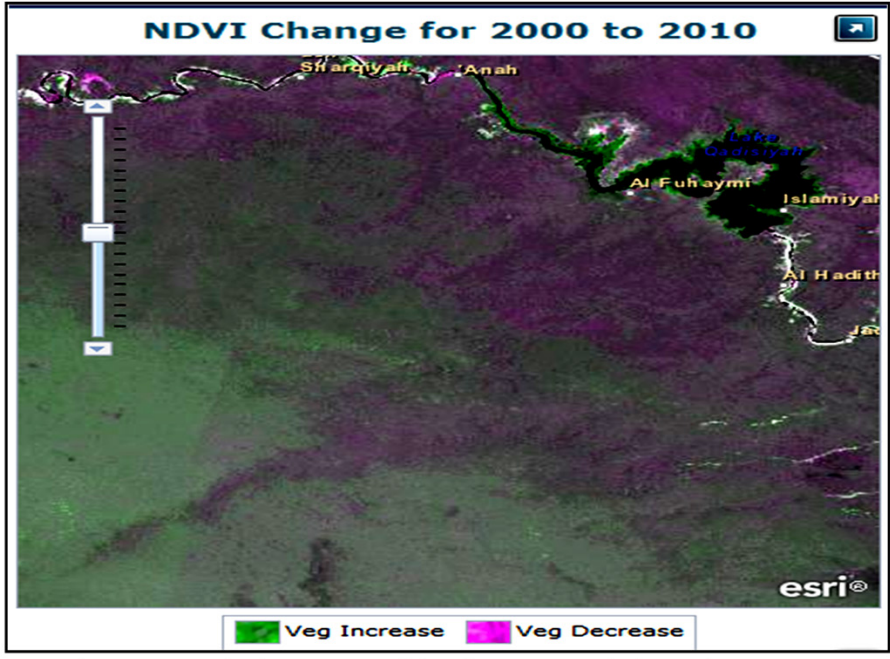

Figure 13. The low area of land cover, and the color green represents the amount of increase in the size of its area 


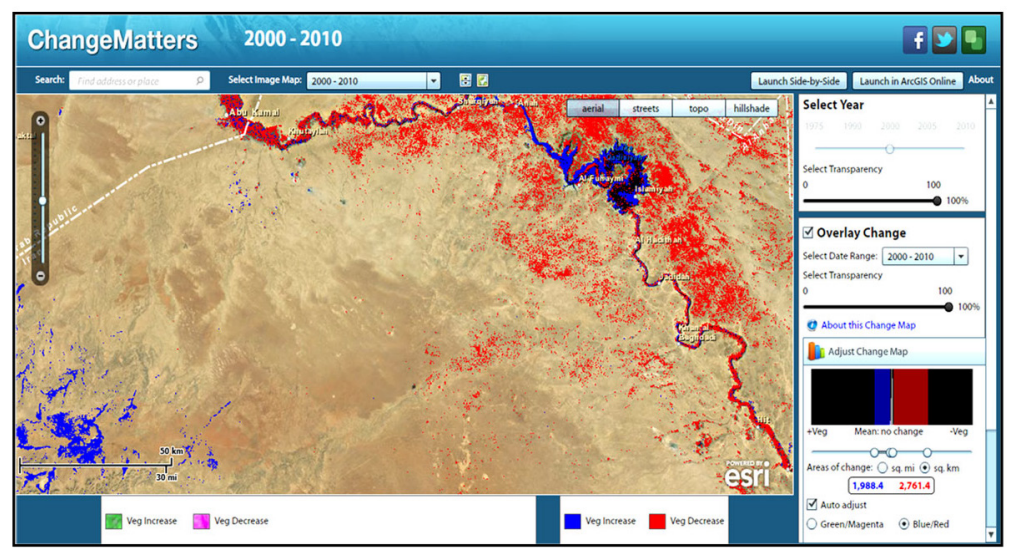

Figure 14. The results of the integration of maps and analysis and after the amendment

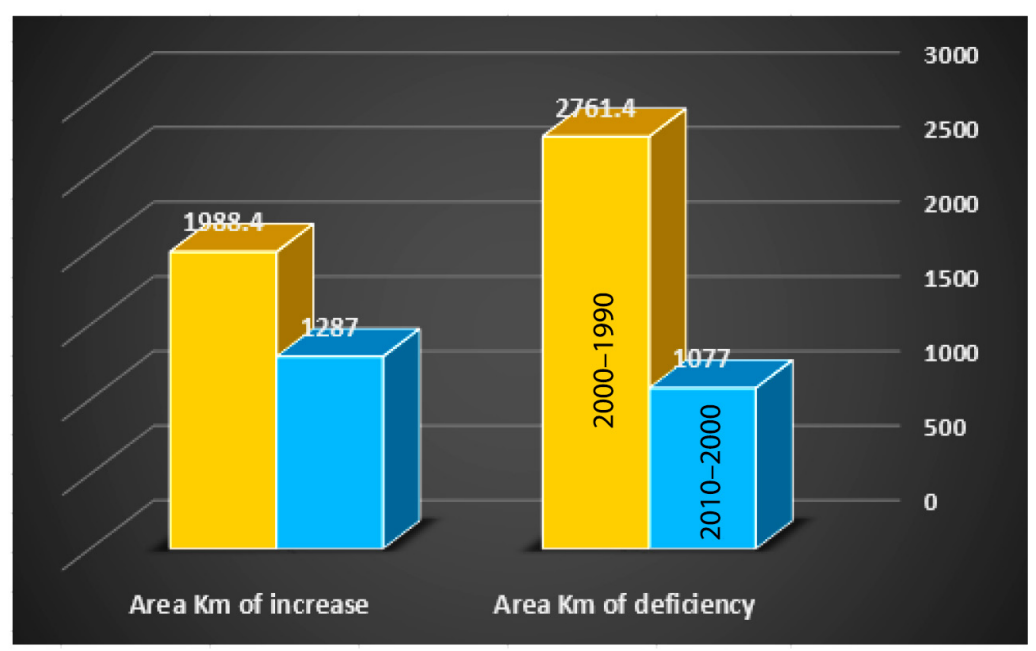

Figure 15. Change in the area of land cover in the research area for the period (1990-2010)

The results of the integration of maps and analysis and after the amendment of the colors red represents the lack of area of land cover, which surrounds the Euphrates River and Lake Qadisiyah, area $2761.4 \mathrm{~km} 2$ by $13.393 \%$, color blue represents the increase area $1988.49 .644 \%$ (Figure 14).

The land cover changes in the search area can be monitored from the derived maps through the analyzes for the years 1990-2010 and Table 1, Figure 15.

Table 1. Area of land cover and percentage of total area for the period (1990-2010)

\begin{tabular}{|c|c|c|c|c|}
\hline$\%$ & $\begin{array}{c}\text { AreaKm of } \\
\text { increase }\end{array}$ & $\%$ & $\begin{array}{c}\text { AreaKm of } \\
\text { deficiency }\end{array}$ & Years \\
\hline 6.24 & 1287 & 5.22 & 1077 & $2000-1990$ \\
\hline 9.64 & 1988.4 & 13.39 & 2761.4 & $2010-2000$ \\
\hline
\end{tabular}

\section{Conclusions}

When working with massive datasets, like Landsat, one of the obstacles encountered early on is how to make the data accessible to users in an easily digestible format. People working with Landsat want to be able to find the information they are looking for; extract the information; and move on to their work, which involves solving some of the most complex problems affecting our society today. There are two Landsat viewers available online that help users do just that - the LandsatLook Viewer from USGS and the ChangeMatters Viewer from Esri. The second viewer available on the web today is the Esri ChangeMatters Viewer. This is a powerful yet easy-to-use web application that provides simple access to the entire Landsat Global Land Survey (GLS). The GLS datasets are collections of the best cloud-free Landsat scenes from the five epochs, circa 1975, 1990, 2000, 2005, and 2010. The ChangeMatters Viewer uses ArcGIS as the underlying technology to additionally process the Landsat GLS dataset on the fly into multiple data products that are served as the World Landsat These web services make all the Landsat data quickly accessible with the ArcGIS Online server providing all the image processing. The ChangeMatters Viewer makes it easy to understand global earth changes in vegetation, urban growth, deforestation, natural disasters, etc. The ChangeMatters Viewer also includes a simple 
Normalized Difference Vegetation Index analysis that calculates the change difference in each pixel from one epoch to another. This is displayed on the screen in two shades, green and magenta, which represent vegetation increase and vegetation decrease, respectively.

View imagery from different years side by side and compare the vegetative change.You will notice that the left and middle panels display two different years. You can select which two years you want to compare by using the Select Years or Dates (depending on browser used) dropdown at the top of the page. The choices include 1975 and 1990, 1975 and 2000, 1975 and 2005, 1990 and 2000, 1990 and 2005, and 2000 and 2005.

This application allows users to get more information about the image maps they are viewing (in this case, "Healthy Vegetation" aka a "4-5-1" in Remote Sensing Speak), as well as so some on-the-fly histogram based change mapping.

The ChangeMatters Viewer also includes a simple Normalized Difference Vegetation Index analysis that calculates the change difference in each pixel from one epoch to another. This is displayed on the screen in two shades, green and magenta, which represent vegetation increase and vegetation decrease, respectively. An easy way to get started with the ChangeMatters Viewer is to watch the ChangeMatters Tips tour. This is a live, interactive tour that provides tips for using the ChangeMatters Viewer to understand the Landsat data. Esri has also applied the new ArcGIS 10.1 advanced imagery processing, correction, and enhancement tools to the web services used in the ChangeMatters Viewer. These new tools include the apparent reflectance function, which provides radiometric corrections to the displayed imagery; raster functions that can be applied to the 1975 epoch to simulate color views of the earth from the Landsat Multispectral Scanner series data by utilizing a virtual blue band; and the ability to automatically destripe scenes that contain the Scan Line Corrector problem, which resulted in data gaps.

\section{Conflict of interest}

I, the original author of the paper, admit that there is no conflict of interest with others in any field.

\section{Funding}

There is no agency, person, or institution to fund the research.

\section{References}

Advanceduninstaller.com. (2008). https://www.advanceduninstaller.com/Universal-Maps-Downloader-6_71-eece492fd90a1e97c6fe1921e8b98967-application.htm

Al-Khuzami, A. M. (2007). Applied studies in GIS. Dar Al-Alam. Al-Din, M. W. (2008). The basics of geographical information systems. Riyadh.

arabspatial.org. (n.d.). http://www.arabspatial.org/

ChangeMatters. (2013). www.changematters.com

DeMers, M. N. (2009). GIS for dummies. Wiley Publishing, Inc.

Esri.com. (2012). https://www.esri.com

gnu.org. (2008). https://www.gnu.org/licenses/gpl-3.0.html

Kropla, B. (2005). Beginning MapServer: Open source GIS development. Apress.

NASA (n.d.). http://www.nasa.gov/

USGS. (n.d.). https://www.usgs.gov/

Wise, S., \& Craglia, M. (2008). GIS and evidence-based policy making. CRC Press. https://doi.org/10.1201/9781420008234

\section{APPENDIX}

${ }^{*}$ SDTS: Spatial Data Transfer Standard.

FGDC: Federal Geographic Data Committee.

ISO: International Standards Organization.

CGIAR: Consultative Group on International Agricultural Research.

Learn more about open source sites: http://maps4pc.com/download/ http://resources.arcgis.com/en/communities/city-engine/ http://www.mapsgalaxy.com/ alreadyInstalled.jhtml http://store.scrapbook.com http://www.arabspatial.org / http://wikimapia.org 